\title{
Intracapillary glomerular metastases in a nephrectomy specimen removed for ipsilateral renal cell carcinoma
}

\author{
R A Carr, A K H Yoong, J Newman
}

\begin{abstract}
A case of intraglomerular metastases observed in a nephrectomy specimen removed for primary renal cell carcinoma is reported. The intraglomerular metastases arose by dissemination of malignant cells into the systemic circulation via invasion of the renal veins. Intraglomerular metastases are therefore an indicator of malignant dissemination which in turn should be associated with a poor prognosis. It is recommended that in nephrectomies undertaken for primary renal cell carcinoma at least one random block of renal cortex should be examined to confirm or exclude intraglomerular metastases.
\end{abstract}

(F Clin Pathol 1994;47:558-559)

In a necropsy series intraglomerular metastases were observed in $7 \%$ of patients who died with metastatic extra-renal carcinomas. ${ }^{1}$ As far as we are aware, there are only two previous case reports of intraglomerular metastases in association with renal cell carcinoma ${ }^{23}$ and only one previous antemortem report of intraglomerular metastases from all types of primary carcinomas. ${ }^{4}$

\section{Case report}

A 52 year old woman presented with an eight week history of intermittent right upper

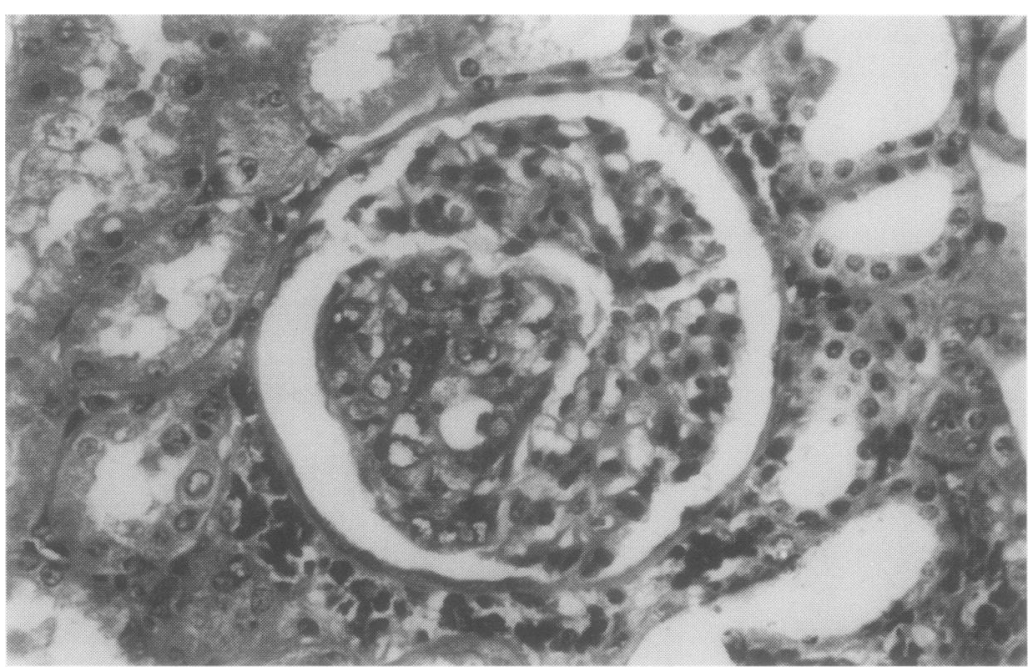

A glomerulus showing an intracapillary metastasis within the left lower segment. A periglomerular lymphoid infiltrate can be seen (haematoxylin and eosin). quadrant abdominal pain which radiated to the loin. At laparotomy an unsuspected right renal mass was identified and a nephrectomy performed. Following the nephrectomy a technetium bone scan showed several foci consistent with metastatic malignancy. She died six weeks after the nephrectomy; a necropsy was not performed.

A right kidney measuring $12 \times 9 \times 8.5 \mathrm{~cm}$ with a lobulated $7 \mathrm{~cm}$ tumour replacing the lower pole, with local invasion through the renal capsule into the perinephric fat, was received. Microscopic examination showed a predominantly moderate to poorly differentiated adenocarcinoma with foci of moderate to well differentiated tubular clear cell pattern and also foci of poorly differentiated sarcomatoid pattern. At the hilum renal vein invasion was seen, but the ultimate vascular pedicle and pelvicalyceal system were free of tumour.

In the renal cortex adjacent to the primary tumour occasional periglomerular lymphoid aggregates were seen in association with intracapillary glomerular metastases (figure). A further 30 blocks were sampled from the remaining renal parenchyma, each having more than 100 glomeruli per section. One section with 130 glomeruli contained no definite intraglomerular metastases; of the remaining 29 sections, intracapillary glomerular metastases were seen widely distributed in $2 \%-6 \%$ of glomeruli. In total 11329 glomeruli were counted with 371 (3.3\%) containing intracapillary glomerular metastases. Occasional sections contained interstitial metastatic deposits. A further 10 blocks were sampled from the hilar parenchyma which showed extensive tumour within vessels of venous calibre but no evidence of arterial invasion.

\section{Discussion}

Two types of intraglomerular metastases have been reported before. ${ }^{1}$ The intracapillary type involves the glomerular tuft as in our case. In the extracapillary type the malignant cells occupy Bowman's space and may mimic epithelial crescents. ${ }^{5}$ Toth's observation of intraglomerular metastases in $7 \%$ of necropsies carried out for extrarenal carcinomas indicates that the true incidence of this phenomenon is greater than that suggested by the paucity of published reports. ${ }^{1}$ However, prior to the present case there is only one previous antemortem report of intraglomerular metastases. This was a renal biopsy performed for renal failure in which all the 
glomeruli contained intracapillary metastases, although the primary origin was not established. ${ }^{4}$

Renal cell carcinomas are multiple or bilateral in up to $20 \%$ of cases. ${ }^{6}$ One might therefore expect intraglomerular metastases to be a common observation. Despite this, intraglomerular metastases have been reported only twice before in association with renal cell carcinoma. ${ }^{23}$ Lisa mentions a single intracapillary glomerular metastasis in a necropsy report of a multicentric bilateral renal cell carcinoma. ${ }^{2}$ The case described by Galloway and Ray is particularly interesting in that the patient developed renal failure a few weeks after a nephrectomy for renal cell carcinoma. At necropsy $80 \%$ of the glomeruli in the remaining kidney contained intracapillary glomerular metastases. ${ }^{3}$

In our case the presence of periglomerular lymphoid aggregates drew attention to some of the affected glomeruli at low magnification. Once observed, further intraglomerular metastases were easily seen on routine haematoxylin and eosin stained sections and at relatively low magnification $(\times 100)$.

There are three possible bloodborne mechanisms by which intraglomerular metastases may arise, firstly by retrograde spread from the renal vein. This route would seem improbable as we would expect extensive permeation of small vessels and interstitial deposits arising from the arrest of malignant cells in the interstitial capillaries before they reach the glomerulus. Extensive sampling revealed a paucity of interstitial metastatic deposits. The second possible route is via invasion of a renal artery. The wide distribution of metastases in our case would require invasion of a main renal artery which was not seen macroscopically or microscopically, despite thorough sampling of the renal hilum. Major arterial invasion might also be expected to have resulted in certain observable sequelae such as haemorrhage or infarction which were not seen. The third route is by dissemination of malignant cells into the systemic venous circulation via invasion of renal veins with passage through the lungs to return to the kidney via the systemic arterial circulation. The wide, uniform, distribution of intraglomerular metastases and extensive involvement of the renal veins observed in our case are in keeping with this last mechanism.

If intraglomerular metastases do indeed occur via dissemination of malignant cells into the systemic circulation then a poor prognosis would be expected. In this case the patient's survival was only six weeks. We therefore recommend that in nephrectomies for renal cell carcinoma the cortex should be examined to confirm or exclude intraglomerular metastases.

1 Toth T. Extracapillary tumourous metastatic crescents in glomeruli of the kidney. Path Res Pract 1987;182:240-3. 2 Lisa JR. Multicentric bilateral carcinoma of the kidneys. Am $\mathcal{f}$ Pathol 1944;21:383-5.

3 Galloway CN, Ray CT. Diffuse glomerular metastases from hypernephroma. Arch Int Med 1964;114:803-5.

4 Belghiti D, Bernaudin JF, Pariente EA, Martin N. Intraglomerular metastases: report of two cases. Cancer 1984;54:2309-12.

5 Ross L. Bronchogenic squamous cell carcinoma metastasizing to Bowman's capsule. F Clin Pathol 1966;19: 375-7.

6 Harvey NA. Kidney tumours: clinical and pathological study with special reference to hypernephroid tumour. f Urol 1947;57:669-92. 